\title{
Double Nail in Hypopharynx- A Rare Presentation
}

\author{
Pranabashish Banerjee, ${ }^{1}$ Rajesh Kumar Kundu, ${ }^{1}$ Dhrupad Ray, ${ }^{1}$ Gautam Das ${ }^{1}$
}

\section{Introduction}

\section{ABSTRACT}

An extremely rare case of a pair of nails impacted in the right pyriform sinus, presenting 72 hours after the incident, is reported. Case Report

$X$-ray soft tissue neck revealed the presence of a pair of nails in the neck at C4-C5 vertebral level. The foreign bodies could not be seen on hypopharyngoscopy due to severe mucosal oedema. Lateral pharyngotomy was done to remove the nails with C-arm guidance.

Discussion

Retained foreign bodies in the aerodigestive tract are known to produce complications. Delayed presentation makes the management difficult. C-arm guidance during surgical exploration is helpful to locate a radiopaue foreign body in difficult situations.

$\underline{\text { Keywords }}$

Foreign Bodies/surgery; Nails; Hypopharynx; Pyriform Sinus

$\mathrm{F}$ oreign body in throat is common emergency encountered in routine otorhinolaryngology practice. Foreign body which we commonly encounter in the upper aerodigestive tract include metallic i.e. coin, pin, needles, safety pin, battery and non -metallic i.e. fish bone, meat bone, chicken bone, artificial dentures, plastic toys etc. There are very few reported cases of foreign body in the hypopharynx. Pharyngeal foreign bodies get lodged in the mucosa and may not be visible on routine clinical examination. ${ }^{1}$

We present here an extremely rare case of a pair of nails impacted in the right pyriform sinus presenting 72 hours after the incident at the emergency department. The absence of ENT facility at the remote residential location in Nepal and absence of acute symptoms were responsible for the delayed presentation.

1 - Department of ENT, North Bengal Medical College, West Bengal

\section{Corresponding author:}

Dr Pranabashish Banerjee

email: drpranab99@gmail.com

\section{Case Report}

A 25 years old male patient presented at otorhinolaryngology emergency with history of accidental ingestion of nails while doing decorative work. Patient had chief complaint of foreign body sensation in throat along with pain during swallowing. There was no history of fever or vomiting. Loco regional examination by indirect laryngoscopy was suggestive of edematous right pyriform sinus with significant pooling of saliva. Laryngeal crepitus was impaired. Laryngeal framework was tender on palpation.

$X$ ray soft tissue of neck - anteroposterior and lateral view was suggestive of a pair of nails impacted in the right pyriform sinus with the sharp end of the nails projecting towards the vertebrae at the level of C4-C5. (Fig.1) We planned to remove the foreign body by hypopharyngoscopy or esophagoscopy under General Anaesthesia .

After proper consent, antiseptic dressing and draping with proper patient positioning, the hypopharyngoscope was introduced. However, due to extremely edematous hypopharyngeal mucosa, foreign body could not be visualised. Per-operative $\mathrm{C}$ arm was used to see the 
exact topographical location of the foreign body and found to be situated at the level of thyroid cartilage. (Fig. 2) However, the foreign body could not be grasped by alligator forceps due to edematous mucosa. Hence, we decided to explore the neck by lateral pharyngotomy approach.

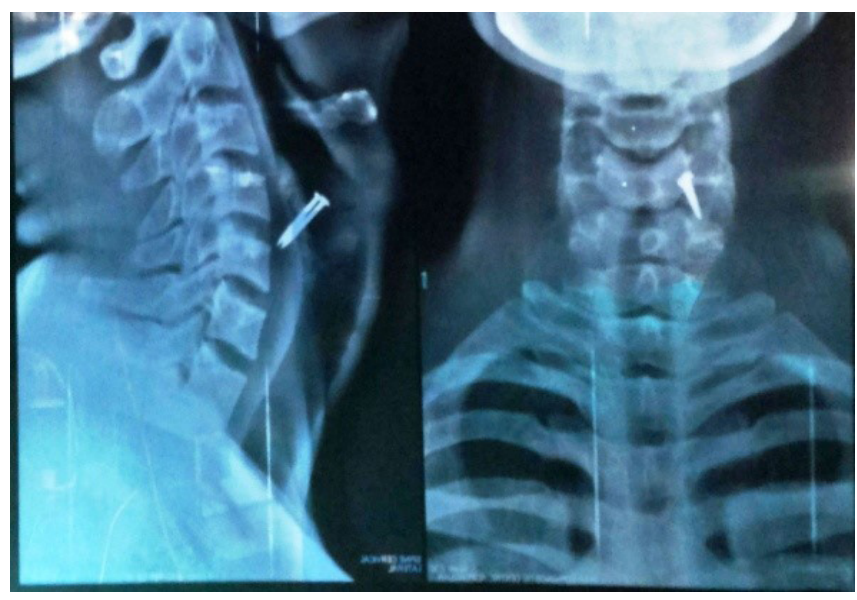

Fig.1. X ray soft tissue of neck - antero posterior and lateral views

Transverse incision was made at the level of upper border of thyroid cartilage. Subcutaneous tissues and platysma were incised along the line of incision. Strap muscles were retracted and the right lateral border

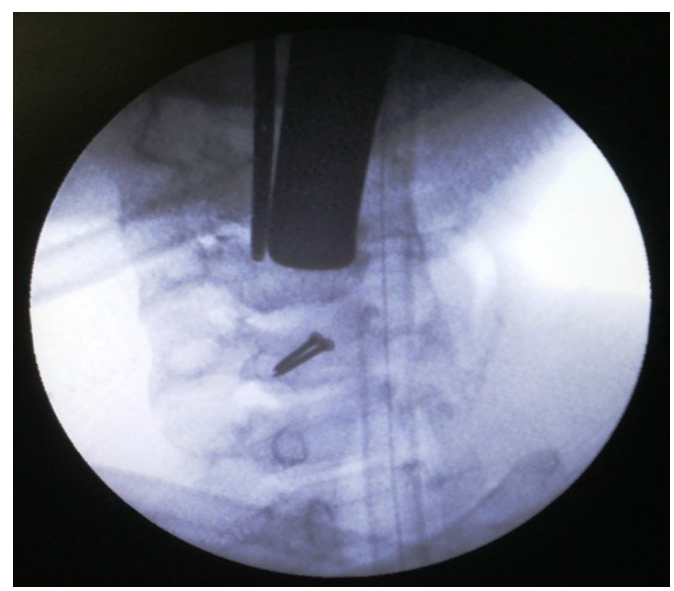

Fig.2. Per operative localisation using $\mathrm{C}$ arm

of thyroid cartilage was delineated. $\mathrm{C}$ arm was used to precisely identify the location of foreign body. Pyriform sinus mucosa was incised just behind the right lateral border of thyroid cartilage close to the superior cornu. Foreign body was identified and gently removed without any inadvertent injury to the adjacent mucosa.(Fig. 3) Nasogastric tube (16 FG) was introduced before pharyngeal mucosal repair. Mucosa was repaired using 3-0 monofilament suture. Corrugated

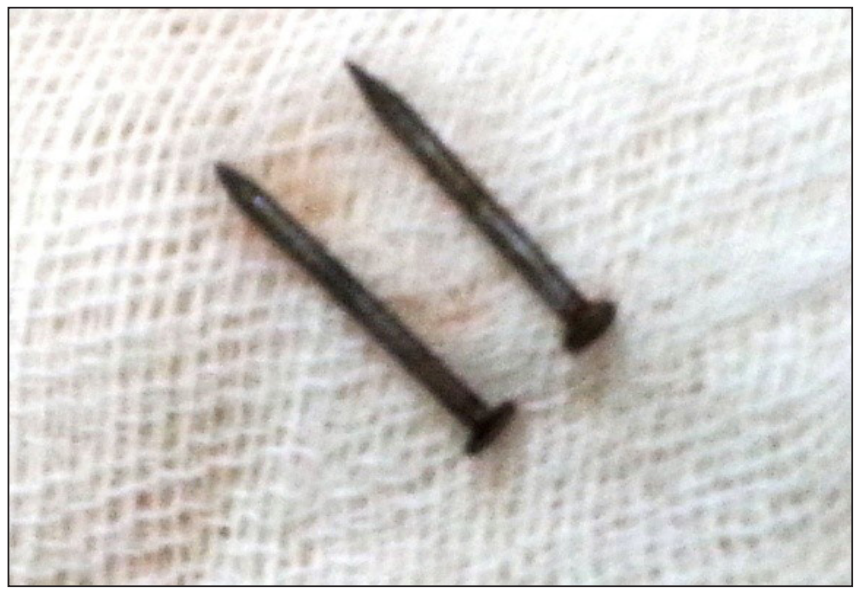

Fig.3. Foreign bodies after removal

rubber drain was placed and the wound was repaired in two layers. The patient had subsequent uneventful recovery. (Fig. 4)

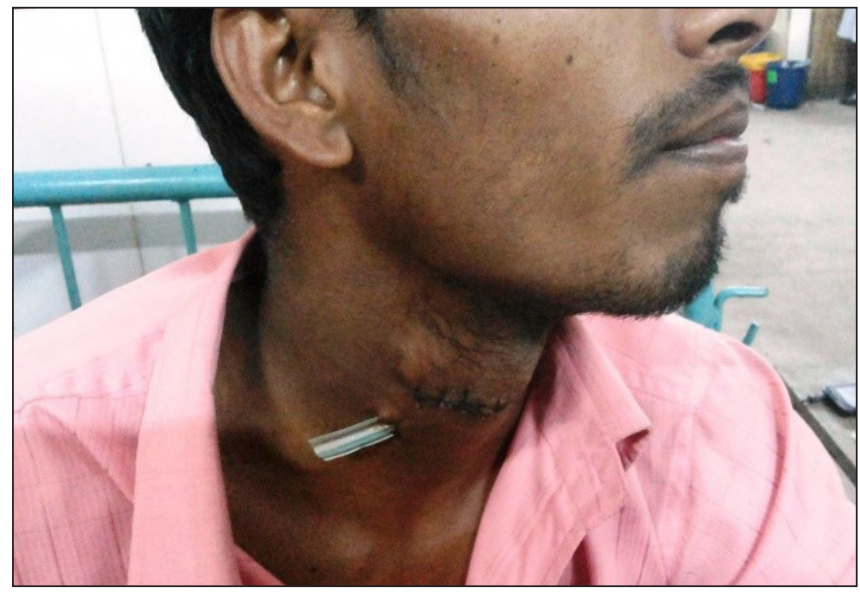

Fig.4. Patient in the post-operative period

\section{Discussion}

Accidental ingestion of foreign body is a common 
emergency in ENT practice. Swallowing of foreign bodies occurs more commonly in children, especially between the age of 6 months and 3 years, and in specific adult risk groups, such as prisoners, alcoholics, edentulous adults and psychiatric patients. ${ }^{2-4}$ Our patient did not belong to any of these risk categories.

History of ingestion of foreign body, inability to swallow saliva and dysphagia are most important symptoms with which a patient usually presents to the Out Patient Department. ${ }^{5}$ In children however, many are asymptomatic. Our patient presented with history of definite ingestion of foreign body along with foreign body sensation in the throat. Usually ingested foreign bodies pass harmlessly through the gastrointestinal tract but a few become impacted at various levels of pharyngeal soft tissue ${ }^{6}$ or oesophagus depending upon the nature of foreign body and obstruction in the oesophagus which may be physiological or pathological like pre existing stenosis or any undiagnosed growth.

$\mathrm{X}$ ray soft tissue neck-lateral and Anteroposterior views are the preliminary radiological investigations which are mandatory even with the slightest suspicion of a foreign body. If the impacted foreign body is radiolucent, in the presence of positive history, symptoms or clinical suspicion, Computed Tomographic scan of neck and thorax and endoscopic examination may sometimes be essential. In most of the cases no medical or surgical intervention is needed. Endoscopic treatment and surgical intervention are necessary in $20 \%$ and $1 \%$ of cases, respectively. ${ }^{7-9}$

In our case, X-ray soft tissue neck- anteroposterior and lateral views revealed two radiopaque sharp tipped linear parallel shadows, each approximately $1.5 \mathrm{~cm}$ in length at the level of $\mathrm{C} 4-\mathrm{C} 5$ vertebrae. There was no coexisting retropharyngeal collection.

Hypopharyngoscopy or oesophagoscopy is usually done for removal of foreign body under general anaesthesia. Rarely, it is extremely difficult to retrieve the foreign body by endoscopic approach as in our case. Excessive mucosal oedema and congestion causing poor localization of the foreign body by endoscopy may be due to prolong stay of foreign body due to delay in presentation of the case. Sharp impact foreign bodies are extremely notorious in this account due to their potential capacity for migration. External approach by lateral pharyngotomy is well established in the literature in such cases. Since the foreign body was very close to the vertebra, we hence decided to remove it by lateral pharyngotomy. C-arm was used to locate the foreign bodies precisely and hence minimizing the chances of injury during their removal.

Some important complications due to neglected foreign bodies of upper aero-digestive tract include scratches and lacerations of oropharyngeal mucosa, perforation, retropharyngeal abscess, soft-tissue infection or abscess, oesophageal perforation and subsequent para-oesophageal abscess, oesophageal necrosis, oesophageal stricture, migration of foreign body, mediastinitis, pneumothorax and/or pneumomediastinum, pericarditis/cardiac tamponade, tracheo-oesophageal fistula, aorto-oesophageal fistulae or other mediastinal vascular injury etc. Considering these myriad and dangerous complications, urgent removal of foreign body is absolutely mandatory.

It is thus always mandatory to give due respect and care to the complaints of the patient and do a proper ear, nose and throat examination, get the investigations done as needed so as not to under diagnose the case or leave a foreign body and give constant irritation and misery to the patient. ${ }^{10}$ Under-diagnosis and delay in presentation may increase the complications and fatality of the cases.

\section{References}

1. Arora S, Sharma JK, Pippal SK, Sethi Y et ai. An unusual foreign body (sewing needle tip) in the tonsils. Braz J Otorhinolaryngol. (Impr.) 2009; 75(6):908

2. Al-Qudah A, Daradkeh S, Abu-Khalaf M. Esophageal foreign bodies. Eur J Cardio-Thoracic Surg. 1998; 13:494-9

3. Cangir AK, Tug T, Okten I. An unusual foreign body in the esophagus: Report of a case. Surg Today 2002; 32:523-4

4. Passali D, Morra B. Upper aerodigestive tract foreign body injury prevention: an ENT evidence-based perspective. Acta Otorhinolaryngol Ital. 2005; 25(Suppl):78

5. Ong NP. Foreign body in Oesophagus: Review of 2394 cases. Br J Surg. 1978; 65:5-9

6. Hachimi-Idrissi S, Corne L, Vandenplas Y. Management of ingested foreign bodies in childhood: our experience and review of the literature. Eur J Emerg Med.1998; 5:319-23 
7. De Lucas EM, Ruiz-Delgado ML, Garcia-Baron PL, Sadaba P, Pagola MA. Foreign esophageal body impaction: multimodality imaging diagnosis. Emerg Radiol. 2004; 10:216-7

8. Furihata M, Tagaya N, Furihata T, Kubota K. Laparoscopic removal of an intragastric foreign body with endoscopic assistance. Surg Laparosc Endosc Percutan Tech. 2004; $14: 234-7$
9. Athanassiadi K, Gerazounis M, Metaxas E, Kalantzi N. Management of esophageal foreign bodies: a retrospective review of 400 cases. Eur J Cardio-Thoracic Surg. 2002; 21:653-6

10. Shinde KJ, Gupta A. An Unusual foreign body in Oesophagus. Indian J Otolaryngol Head Neck Surg 1999; 7B:62-4 